\title{
Constrained vs spontaneous speech and gestures for interacting with computers: A comparative empirical study
}

\author{
Sandrine Robbe*, Noëlle Carbonell*, Pierre Dauchy** \\ *CRIN-CNRS \& INRIA-Lorraine, BP 239, F54506 Vandœuvre lès Nancy cedex \\ **IMASSA-CERMA, BP 73, F91223 Brétigny sur Orge cedex \\ robbe@loria.fr, carbo@loria.fr pierre@cerma.fr
}

\begin{abstract}
In the framework of a prospective ergonomic approach, we simulated two multimodal user interfaces in order to study the usability of constrained vs spontaneous speech. The first experiment, which served as a reference, gave subjects the opportunity to use speech and gestures freely. Contrastingly, in the second experiment, subjects had to comply with multimodal constraints. We conducted a comparative study between these two experiments which aims to provide designers with useful knowledge concerning the requirements, preferences and expectations of potential users of future multimodal interfaces. The paper presents both experiments together with the conclusions of our analyses of the collected data.
\end{abstract}

KEYWORDS Design rationale, Interaction design, Speech and voice, Usability evaluation, Multimodal user interfaces

\section{INTRODUCTION}

Fuller transparency of Human-Computer Interaction (HCI) ranks among users' major expectations; in particular, it is much sought after by occasional users and the general public, inasmuch as the cognitive cost of learning an artificial language is higher for them than for users who intend to become experts or frequent users (Shneiderman, 1987).

Thanks to recent advances in both automatic continuous speech recognition and natural language (NL) interpretation, it is now possible to process large subsets of spoken NL accurately, and to substitute them for existing artificial query or command languages (Seneff et al., 1996), with a view to offering users "natural" (hence transparent) HCI. Nevertheless, researchers in computational linguistics have not yet reached a thorough understanding of spatial reference phrases and anaphora mechanisms. Thus, the implementation of oral NL as a substitute for direct manipulation (Shneiderman, 1983) cannot yet be considered, since the control of iconic interfaces requires the ability to indicate locations on the screen, and to select a specific icon from a set of similar graphical objects. An attractive solution for achieving robust quasi"natural" $\mathrm{HCI}$ soon is to design $\mathrm{HCI}$ languages that include, as well as oral statements from a given subset $\mathrm{S}$ of $\mathrm{NL}$, multimodal ${ }^{1}$ commands associating oral statements from $S$ with pointing gestures. Research on the implementation of multimodal $\mathrm{HCI}$ is developing rapidly ((Bellalem and Romary, 1995) (Nigay and Coutaz, 1993)). In addition, field studies (Falzon, 1991) indicate that operators spontaneously develop specific operative sublanguages for professional exchanges; these languages consist of subsets of natural language with a restricted syntax and a limited nonambiguous vocabulary.

We are currently investigating, within a prospective ergonomic research framework, utility and usability issues pertaining to the implementation of this short-term ap-

\footnotetext{
'Subsequently, "multimodality" and "multimodal" will refer to the association of speech and gestures only.
} 
proach. The main goal of the comparative empirical study reported here is to assess the influence of realistic expression constraints on the behaviours of potential users of future multimodal interfaces. More precisely, we address the following issues, with a view to providing designers with useful knowledge concerning the requirements, preferences and expectations of multimodal interface users:

- Is it possible to define realistic constraints on users' spontaneous forms of multimodal expression, which they will comply with easily, as well as learn rapidly and reliably (mostly through the interaction)?

- What is the influence of such constraints (especially system recognition failures) on users' activities, their use of multimodality, and the efficiency of the interaction?

- Which design methods will guarantee the generation of "good" expression constraints, that is: constraints which are easy to apply, do not interfere with users' activities, and maintain the efficiency and the appeal of the interaction?

To answer these questions, we considered two experimental situations: in the first, which served as a reference, subjects could use speech and gestures freely, whilst in the second they had to comply with multimodal constraints.

We first describe the experimental setup. Then we analyse and interpret the resulting empirical data. Finally, we summarize our main results from which we infer useful conclusions for the design of multimodal HCI.

Whilst constrained oral HCI has motivated numerous experimental or empirical studies (Hauptmann and McAvinney, 1993), few empirical studies have been devoted to multimodal constrained HCI (Catinis and Caelen, 1995); and, to our knowledge, no comparative study has been published thus far. The issues we mentioned earlier have not yet been addressed.

\section{EXPERIMENTAL SETUP}

\subsection{Overview}

Two groups, of eight subjects each, interacted, during three weekly sessions, with two different multimodal interfaces whose functionalities were simulated thanks to the Wizard of $\mathrm{Oz}$ technique. The average length of a session was about half an hour (per subject). Both groups carried out identical tasks; but subjects in the reference group (SP) could use speech and/or 2D gestures spontaneously, while subjects in the experimental group (CS) had to comply with expression constraints.

Although human face-to-face communication involves 3D gestures more often than 2D gestures, we restricted the nonverbal expression of subjects to $2 \mathrm{D}$ gestures i.e. gestures on the surface of the screen - in order to offer them a realistic HCI environment, that is, a user interface that could be implemented in the near future. In addition, as application objects were represented by $2 \mathrm{D}$ icons, gestures in a 3D space were not needed.

The application domain and the tasks that subjects had to perform were chosen so that all the necessary actions for achieving the scenarios could be expressed easily using just speech, or just gestures, or a combination of both modalities.

\subsection{Expression constraints}

In order to obtain a tractable multimodal artificial language that would impose minimal constraints on the spontaneous expression of CS subjects, we selected an appropriate subset of the overall set of utterances and gestures used by SP subjects. The gestural component of this language included two categories of elementary gestures: pointing gestures (PG) and simulation gestures (SG), that is, gestures (akin to mouse drags) that mimed requested translations or rotations of graphical objects. Ambiguous gestures and constructs, and no others, were eliminated from the simple gestural "vocabulary" and "syntax" used by SP subjects.

We adopted the following selection criteria for defining the oral component of the language:

- Utterances should be reliably interpretable using current continuous speech recognisers; therefore, the size of the vocabulary was restricted to a hundred lexical items or so, and the syntax was simplified so that it could be described by a CF grammar (the static branching factor of this grammar is 5.5 and its dynamic branching factor is 2.6);

- The language expressiveness (i.e. its semantics) should be equivalent to the union of the semantic interpretations of all the utterances issued by SP subjects over the three sessions - we wanted to sepa- 


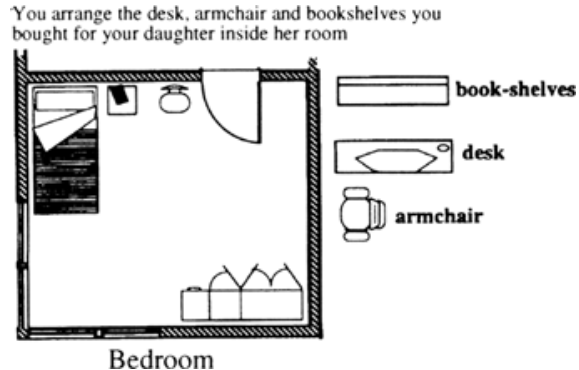

(A)

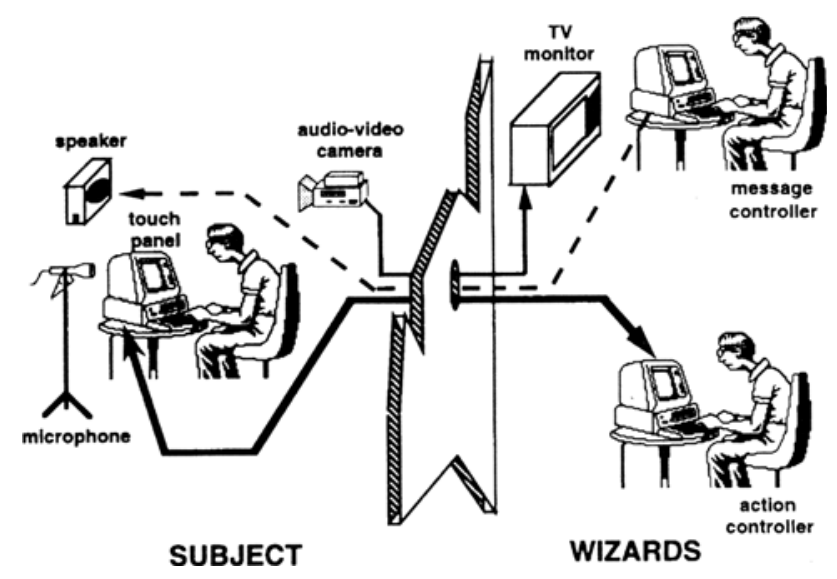

(B)

Figure 1: (A) First scenario: text and initial display. (B) Experimental setup.

rate the possible effects of lexical and syntactic constraints on the expression and activity of CS subjects from the influence of semantic restrictions, so that the effects of lexical and syntaxic constraints could be assessed accurately through a comparison of CS and SP subjects' behaviours;

- Synonyms and ambiguities (at the lexical and syntactic levels) should be eliminated, as they are excluded from operative sublanguages consisting of subsets of NL defined spontaneously by operators.

CS subjects were given a written description of this multimodal artificial language (MAL), and the experimenter helped them to perform the examples included in this short manual before the execution of the first scenario; this initial training stage lasted less than 10 minutes on average. SP subjects had no initial training, but they could, before launching the first scenario, explore the capabilities of the interface in the presence of the experimenter who just answered their questions.

\subsection{Subjects}

All subjects had previously used software intended for the general public, and most of them were familiar with direct manipulation; some had basic programming knowledge, but none was an expert in computer science. Their backgrounds and professions were similar.

\subsection{Application domain and tasks}

Subjects were asked to perform design tasks relating to furniture arrangement. They had to modify the layout of various furnished rooms according to instructions specified in scenarios of increasing complexity. Initial layouts were displayed on the screen in the form of 2D plans. Figure 1(A) reproduces the first scenario and the corresponding initial layout.

\subsection{Implementation of the WOZ technique}

Two human operators were in charge of the simulation of the functionalities of both multimodal interfaces.

One of them interpreted the subject's commands and activated the relevant software functions; results were displayed simultaneously on the subject's and the wizards' screens. The other operator, who was taking care of dialogue management, selected and activated pre-recorded oral messages in response to incoming utterances and gestures from the subjects. The experimental setup is described in Figure 1(B).

In addition, the CS setup included a commercial continuous speech monospeaker recognizer, since the wizards 
could not decide accurately, in real time, whether or not an oral command belonged to the oral component of the MAL language. Wizards interpreted oral utterances from the subjects using the results of the recognition system (which were displayed on the subject's and the wizards' screens).

\subsection{Recordings and transcripts}

Subjects were videotaped throughout both experiments. Written descriptions of the video recordings consist of orthographic transcripts of utterances, and standardized descriptions of subjects' gestures and system actions.

Results of the speech recognizer were included in the transcripts of the CS group.

Transcripts of both first sessions (one scenario) were analysed and compared. Results of the comparisons are summarized in the next section.

\section{RESULTS AND INTERPRETATIONS}

Results, interpretations, discussions and conclusions are grouped according to the issues presented in the introduction.

\subsection{Application of expression constraints}

Subjects in the CS group complied very easily with gestural constraints: we picked out only three "incorrect" gestures in the transcripts. This result is not surprising since subjects were allowed to use only a few gestures based on simple forms.

On the other hand, all subjects resorted to words outside the vocabulary, at least once. Most of these lexical items are semantically related to words in the vocabulary (e.g. by synonymy or antonymy). All subjects corrected some of their lexical errors spontaneously, generally after the first rejection of the corresponding utterances by the system; half of the subjects corrected all of them. Such lexical errors are not frequent, although their frequency varies greatly from one subject to another: the percentages (per subject) of utterances including such errors range from $1 \%$ to $30 \%$, but rates for five subjects are lower than $10 \%$.

Syntactic errors are fewer than lexical ones (23 vs 32 in the whole corpus of transcripts), and only five subjects are concerned. But these errors were detected and corrected less easily ( $30 \%$ vs $56 \%$ ).

On the whole, subjects in the CS group, although they had gone through a very short initial training, managed to confine the oral components of their requests within the boundaries of a restricted subset of NL without apparent difficulty (few errors). As the MAL language can compare with standard command languages, with respect to the vocabulary size and structural complexity, the reasons for this success may be ascribed to specific properties of this artificial language, and thus to the method used for its design, that is:

- MAL is a subset of NL;

- its power of expression has been defined from the analysis of the oral commands and statements uttered spontaneously by SP subjects; it is then equivalent to the power of expression of the spontaneous oral statements formulated by users interacting with the same application, performing similar tasks, and aiming to achieve identical goals;

- synonymy and polysemy (i.e. lexical ambiguity) have been excluded, as well as structural synonymy and ambiguity, in order to emulate the efficiency of operative languages.

Nevertheless, this method has some drawbacks that may preclude its application in industrial environments. The observation of users interacting freely with the application (cf. the SP study) is costly, and the definition of an appropriate but tractable subset of NL may prove tricky ${ }^{2}$. Moreover, the bulk of this investment is application-dependent and then not reusable in other application domains.

An inter-group comparison suggests that subjects from the CS group benefited from the linguistic constraints with which they had to comply. Hesitations and grammatical incorrections are significantly (Wilcoxon test ${ }^{3}$ : $\left.\Sigma_{C S}=43 \mathrm{~W}(8 ; 8)=\right] 49 ; 87[; \mathrm{p}<0.05)$ less frequent in their oral statements than in those from SP subjects: $13 \%$ vs $53 \%$, and $1.9 \%$ vs $23.5 \%$ respectively ${ }^{4}$. These results are detailed in Table 1.

In addition, CS subjects formulate their oral requests more precisely than SP subjects: half of the subjects in

\footnotetext{
${ }^{2}$ For instance, words divide the user's activity into elementary tasks that do not necessarily coincide with the application functions.

${ }^{3}$ We used a Wilcoxon test when there was a significant difference between variances and a t-test in other cases.

${ }^{4}$ Percentages represent numbers of relevant tokens normalized by the total number of oral and multimodal commands per group.
} 


\begin{tabular}{|c|c|c|c|c|c|c|c|c|c|c|c|c|c|c|c|c|}
\hline & \multicolumn{8}{|c|}{ Hesitations } & \multicolumn{8}{|c|}{ Incorrections } \\
\hline & $\mathrm{S} 1$ & S2 & S3 & S4 & S5 & S6 & S7 & S8 & S1 & S2 & S3 & S4 & S5 & S6 & S7 & S8 \\
\hline SP subject & 3.1 & 12.5 & 1.6 & 3.1 & 7.8 & 4.7 & 12.5 & 7.8 & 7.8 & 12.5 & - & - & - & - & 1.6 & 1.6 \\
\hline CS subjects & 2.3 & 1.5 & 1.1 & 0.8 & 1.9 & 1.5 & 3.8 & 3 & 0.4 & - & - & - & - & 0.4 & 0.8 & 0.4 \\
\hline
\end{tabular}

Table 1: Percentages of hesitations and grammatical incorrections over the total number of oral and multimodal utterances per subject $(\mathrm{S} 1, \ldots, \mathrm{S} 8)$

the SP group vs two in the CS group expressed one (or more) imprecise or ambiguous oral request. But it would be rash to draw conclusions from a few instances per group only. Further empirical or experimental studies are needed to evaluate what appears as a mere observed tendency.

This positive influence of the linguistic constraints imposed on CS subjects may be due to the fact that the protocol of the CS study met a request formulated by most SP subjects in the post experiment questionnaires. They stated that they would have greatly appreciated an initial presentation of the interface, including actual instances of commands and interaction sequences. As a justification for this request, they complained that they had been disconcerted, at first, by the new interface they had to test. The attitudes of SP subjects suggest that beginners require illustrated presentations of the capabilities and functioning of new interfaces and $\mathrm{HCI}$ paradigms, even in the case when the new interface or mode of interaction may be viewed as an implementation of the concept of "transparency".

We analyse, in the next section, the possible effects of the expression constraints that we selected on the activity of users, on their use of multimodality and the efficiency of the interaction.

\subsection{Side-effects of expression constraints \\ 3.2.1 On the use of modalities}

There are marked differences between the two groups as regards their use of speech and gestures (cf. table 2). But these differences are not statistically significant by reason of intra-group pronounced inter-individual variations $\left(\Sigma_{C S}=67 \mathrm{~W}(8 ; 8)=\right] 49 ; 87[; \mathrm{p}<0.05)$. The comparison is focused solely on the initial formulations of commands, due to the low number of repetitions and reformulations expressed by SP subjects, whose requests are interpreted by human wizards. Contrastingly, CS subjects are induced to make multiple repetitions and reformulations since their oral statements are interpreted by a commercial speech recognizer with limited accuracy.

According to table 2, the multimodal commands issued by $\mathrm{CS}$ subjects involve pointing gestures (PG) exclusively (MP multimodality), while SP subjects, in the same context, slightly favour the use of simulation ${ }^{5}$ gestures (SG) over PG gestures. This contrast, which is not statistically significant $\left(\Sigma_{C S}=74,5 \mathrm{~W}(8 ; 8)=\right] 49 ; 87[; \mathrm{p}<0.05)$, might derive from differences in the experimental setups. CS subjects were shown instances of both types of multimodal commands; they had then the opportunity to discover the redundancy of multimodal commands involving SG gestures (MS multimodality) and, by comparison, to appreciate the conciseness and accuracy of semantically equivalent MP statements. On the other hand, SP subjects could only rely on their previous experience of direct manipulation to elaborate multimodal commands. This result constitutes an additional argument in favour of the necessity of a minimum initial training, and illustrates the limitations of users' discovery and exploration capabilities.

The fact that CS subjects resort to speech more frequently than SP subjects (cf. table 2) may be viewed as a consequence of their reluctance to use MS formulations, since there is no significant $(t(7,7)=0.0002 ; \mathrm{p}<0.05)$ difference between the two groups as regards the use of gestural (PG or SG) commands.

The use of speech and gestures for expressing reformulations is influenced by factors other than individual preferences, previous experience or the information content of requests. Table 3 describes the modalities selected by CS subjects for reiterating oral requests that the speech recognizer had failed to recognize accurately; repetitions and reformulations of gestural commands are ignored because of their very low frequency ( 23 occurrences, mak-

\footnotetext{
${ }^{5}$ That is, they simulate translations and rotations of pieces of furniture with gestures akin to mouse drags.
} 


\begin{tabular}{|c|c|c|c|c|c|c|c|c|c|c|c|c|c|c|c|c|c|c|c|c|}
\hline & \multicolumn{10}{|c|}{ SP subjects } & \multicolumn{10}{|c|}{ CS subjects } \\
\hline & S1 & S2 & S3 & S4 & S5 & S6 & S7 & S8 & Tot & $\%$ & S1 & S2 & S3 & S4 & S5 & S6 & S7 & S8 & Tot & $\%$ \\
\hline $\mathrm{O}$ & 1 & 2 & 1 & 4 & 3 & 10 & 6 & 2 & 29 & 33 & 37 & 33 & 5 & 1 & 7 & 10 & 15 & 5 & 113 & 49.5 \\
\hline SG & 5 & 7 & 8 & - & - & - & - & 2 & 22 & 25 & 3 & 4 & - & 1 & - & - & 35 & 16 & 59 & 26 \\
\hline PG & 1 & - & 1 & - & - & 1 & - & 9 & 12 & 14 & 23 & 1 & - & 2 & 1 & 1 & 1 & 1 & 30 & 13 \\
\hline MS & 15 & 4 & - & - & - & - & - & - & 19 & 21 & - & - & - & - & - & - & - & 1 & 1 & 0.5 \\
\hline MP & 1 & 2 & - & - & 3 & - & - & - & 6 & 7 & 1 & - & 2 & - & 1 & - & 21 & 1 & 26 & 11 \\
\hline
\end{tabular}

O: Oral commands

SG: Simulation Gestures

PG: Pointing Gestures

M...: Multimodality involving simulation gestures (MS) or pointing gestures (MP)

Tot: Total number of requests per type of modality and per subject

$\%$ : Percentage of $\mathrm{O}$ (or $\mathrm{SG}, \ldots$ ) requests over the total number of requests per group (i.e. SP: $88, \mathrm{CS}: 229$ )

Table 2: Use of modalities (initial formulations of requests)

\begin{tabular}{|c|c|c|c|c|c|c|c|c|c|c|c|c|c|c|c|c|}
\hline & \multicolumn{8}{|c|}{ reformulations } & \multicolumn{8}{|c|}{ final reformulations } \\
\hline & S1 & $\mathrm{S} 2$ & S3 & S4 & S5 & S6 & S7 & S8 & S1 & S2 & S3 & S4 & S5 & S6 & S7 & S8 \\
\hline $\mathrm{O}$ & 9 & 66 & 12 & 1 & 5 & 7 & 8 & 7 & 3 & 13 & 2 & 1 & - & 2 & 3 & 1 \\
\hline SG & 4 & 12 & 1 & - & 1 & 1 & 4 & - & 4 & 12 & 1 & - & 1 & 1 & 4 & - \\
\hline PG & 2 & 2 & 1 & - & 2 & 3 & - & - & 2 & 2 & 1 & - & 2 & 3 & - & - \\
\hline MS & - & - & - & - & - & - & - & - & - & - & - & - & - & - & - & - \\
\hline MP & - & - & 2 & - & 2 & - & - & - & - & - & 2 & - & 1 & - & - & - \\
\hline Tot & 47 & 100 & 21 & 2 & 13 & 18 & 47 & 15 & 47 & 100 & 21 & 2 & 13 & 18 & 47 & 15 \\
\hline
\end{tabular}

Tot: Total number of oral and multimodal requests (initial formulations + reformulations)

Table 3: Use of modalities (reformulations of unrecognized oral requests)

ing up $10 \%$ of all repetitions and reformulations). Results in Table 3 show that six CS subjects resort occasionally to gestural or multimodal expressions after the recognition failure of an oral command, and that the tendency to switch over to SG, PG or MP formulations increases as the number of consecutive recognition failures increases: from $21 \%$ of the intermediate (unsuccessful) reformulations to $59 \%$ of the final (successful) reformulations. Multimodality appears thus as a useful feature for user interfaces that implement unreliable communication media.

Finally, subjects from both groups favour monomodal commands ( $72 \%$ and $88 \%$ of initial formulations, for the SP and the CS groups respectively; cf. table 2) over the simultaneous use of speech and gestures. This observation contradicts a widespread assumption within the relevant scientific community, namely: the naturalness of multimodal commands such as "Put this (+ pointing at an object) here (+ pointing at a location on the screen)". Further empirical or experimental research is needed in order to address this issue which is crucial for the design of future multimodal $\mathrm{HCI}$.

\subsubsection{On the activity and interaction}

Expression constraints may have influenced the activities of the CS subjects to some extent. The comparison between the two groups yields results that are difficult to interpret for the following two main reasons.

Firstly, subjects had to perform design tasks; it is then impossible to evaluate the efficiency of their activities, and to draw conclusions from quantitative cues such as: the global time spent on performing the scenario, the average execution time of the various types of elementary actions, the adequacy of intermediate and final results to 


\begin{tabular}{|c||c|c|c|c|c|c|c|c|}
\cline { 2 - 9 } \multicolumn{1}{c|}{} & S1 & S2 & S3 & S4 & S5 & S6 & S7 & S8 \\
\hline SP subjects & 5.7 & 5.7 & 3.4 & 3.4 & 1.1 & 2.3 & 1.1 & 2.3 \\
\hline CS subjects & 0.5 & 0.5 & 0.5 & - & - & - & 0.9 & 1.7 \\
\hline
\end{tabular}

Table 4: Simple vs complex commands - Percentage of complex requests over the total number of requests (initial formulations)

the requirements expressed in the scenario, etc. Within the framework of design tasks, such cues are either irrelevant or ambiguous. The results presented here should be considered as tentative. In order to assess their reliability and the soundness of our interpretations, it is necessary to compare them with results obtained in the context of other activities.

Secondly, creative tasks promote the expression of idiosyncrasies; this may explain why we observed large inter-individual differences, and obtained no statistically significant result even though we detected group tendencies, that is, behaviour patterns from which one or two subjects only (in the considered group) departed.

CS subjects performed a greater number of actions than SP subjects, but this result is not significant $\left(\Sigma_{C S}=79\right.$; $\mathrm{W}(8 ; 8)=] 49 ; 87[; \mathrm{p}<0.05)$. On the contrary, their actions were significantly $\left(\Sigma_{C S}=38 ; \mathrm{W}(8 ; 8)=\right] 49 ; 87[; \mathrm{p}<0.05)$ less complex than those performed by SP subjects ${ }^{6}$.

The second inter-group difference suggests two plausible interpretations. The first one is based on the following observation that has been reported in several psychological studies of oral human communication (Harris et al., 1980). That is, interlocutors adapt their behaviours to the capabilities and competences of their dialogue partners, the extent of which they infer from a priori knowledge and/or guess from the first dialogue exchanges. The CS subjects decomposed their activities into smaller and simpler units because they inferred from the poor speech understanding capacity of the simulated system that its functional capabilities were also limited. Thus they made no distinction between linguistic and functional limitations in their evaluation of the system competences. Such confusions have been observed in the context of Wizard of $\mathrm{Oz}$ empirical studies of complex oral HCI (cf. (Amalberti et al., 1993) for information di-

\footnotetext{
${ }^{6}$ Subjects could move pieces of furniture and orientate them (simple actions), or combine both types of actions in complex commands such as the permutation of two pieces of furniture, for example: "Put the television set in the North-West corner of the room, facing the settee."
}

alogues).

On the other hand, it might be assumed, within the framework of a cognitive load model, that CS subjects requested the execution of simpler actions as a compensation for the additional cognitive cost of their adaptation to expression constraints; one might conclude then, that expression constraints have a negative side-effect on users' activities inasmuch as they reduce the efficiency of "natural" multimodal HCI.

We also analysed other aspects of the activity of CS and SP subjects: the scope of their action plans, their reactions to irrecoverable communication failures and impossible actions, the strategies they adopted for arranging pieces of furniture in the room, among others. But intergroup comparisons did not reach statistical significance, by reason of the intra-group variability and/or the insufficient amount of relevant tokens.

To summarize, inter-individual differences, together with insufficient empirical data, make it impossible to reach a clear conclusion as regards to the influence of linguistic constraints on the activities of subjects and their use of modalities.

\section{GENERAL CONCLUSION}

We designed, within the framework of a prospective ergonomic approach, two multimodal user interfaces in order to study the usability of constrained vs spontaneous speech and gestures. The functionalities of both interfaces were simulated according to the Wizard of $\mathrm{Oz}$ experimental paradigm. Each interface was used during three weekly sessions by eight subjects who carried out simple design tasks.

Comparative analyses yielded empirical results that suggest useful conclusions for the design of the next generation of multimodal user interfaces for the general public, namely:

- Even drastic linguistic constraints will be well ac- 
cepted and easily complied with by users, provided that the resulting language is a subset of NL which does not impose semantic restrictions on the expression of users. We propose an efficient but costly method for designing such tractable artificial languages in the context of interactive applications involving a restricted pragmatic domain.

- A short initial training period or tutorial is useful for beginners, even with "transparent" interfaces.

- Constraints that satisfy the requirement mentioned in the first alinea may have positive side-effects on users; in particular, they may entail a reduction of hesitations and incorrect statements.

As for the side-effects of expression constraints on users' activities, it is impossible to draw clear conclusions from the empirical data we analysed and compared.

Our results have been obtained from the transcripts and videotapes of the first session (one scenario only). During this session, several SP and CS subjects (one SP, and at least two CS) explored and tested the capabilities of the simulated multimodal interface systematically, while the others just performed the scenario. This may explain why the comparisons between the two groups yielded few significant results. The empirical data collected during the two following sessions should be easier to interpret, since subjects are likely to have given up previous exploration strategies and activities.

Moreover, as behaviours and strategies tend to stabilize under the influence of practice, evolutions during sessions 2 and 3 should be slower than during the first session. Therefore, the effects of expression constraints on subjects' behaviours should be more noticeable during these sessions. A careful analysis, subject-by-subject, of the data collected during sessions 2 and 3 might even contribute to a better understanding of the behaviours of subjects during the first session.

\section{Acknowledgements}

We express our thanks to R. Amalberti and C. Valot, researchers at CERMA, for their significant contributions to the definition and implementation of the experimental setup. This comparative study was partially supported by the French Ministry of Defence (D.G.A./D.R.E.T. contract $\mathrm{N}^{\circ}$ 95-125).

\section{References}

Amalberti, R., Carbonell, N., and Falzon, P. (1993). User representations of computer systems in humancomputer speech interaction. International Journal of Man-Machine Studies, 38:547-566.

Bellalem, N. and Romary, L. (1995). Reference interpretation in a multimodal environment combining speech and gesture. In First International Workshop on Intelligence and Multimodality in Multimedia interfaces, Edinburgh.

Catinis, L. and Caelen, J. (1995). Analyse du comportement multimodal de l'usager humain dans une tâche de dessin. In $I H M$ '95, pages 123-129, Toulouse: Cépaduès.

Falzon, P. (1991). Studying functional dialogues and operative languages. In Queinnec, Y. and Daniellou, F., editors, Designing for everyone. XI Congress of the IEA., Paris. Londres: Taylor and Francis.

Harris, G., Begg, I., and Upfold, D. (1980). On the role of the speaker's expectations in interpersonal communication. Journal of Verbal Learning and Verbal Behavior, 19:597-607.

Hauptmann, H. and McAvinney, P. (1993). Gestures with speech for graphic manipulation. International Journal of Man-Machine Studies, 38:231-249.

Nigay, L. and Coutaz, J. (1993). A design space for multimodal interfaces: concurrent processing and data fusion. In InterCHI'93, pages 172-178, New York: ACM.

Seneff, S., Goddeau, D., Pao, C., and Polifroni, J. (1996). Multimodal discourse modelling in a multi-user multidomain environment. In Fourth International Conference on Spoken Language Processing, pages 1922-195, Philadelphia.

Shneiderman, B. (1983). Direct manipulation: a step beyond programming languages. IEEE computer, 16(8):57-69.

Shneiderman, B. (1987). Designing the user interface: Strategies for effective human-computer interaction. New York: Addison-Wesley Publishing Company. 